\title{
Evaluación genética directa y usando características correlacionadas en ganado Holstein y Jersey Colombiano
}

\author{
Zambrano, J.C. ${ }^{@} ;$ Echeverri, J.J. ${ }^{2}$ y López-Herrera, A. ${ }^{2}$
}

'Fundación Universitaria Navarra. Neiva, Huila, Colombia.

${ }^{2}$ Universidad Nacional de Colombia Sede Medellín. Medellín, Colombia.

\section{PALABRAS CLAVE ADICIONALES}

Exactitud.

Modelo animal.

Valor estimado de cría.

\section{RESUMEN}

El objetivo de esta investigación fue estimar valores genéticos de características reproductivas usando modelos univariados y bivariados en ganado Holstein y Jersey de Antioquia, Colombia. La información fue obtenida de 92 hatos lecheros, ubicados en 18 municipios del Departamento de Antioquia. Las características evaluadas fueron: intervalo entre partos (IEP), días abiertos (DA), número de servicios por concepción (NSC) y tasa de concepción (TC). Los valores estimados de cría (EBV) para características reproductivas fueron calculados mediante dos tipos de modelo animal univariado (modelo univariado, UV y modelo univariado con repetibilidad, UVR) y dos tipos de modelos animal bivariado (modelo bivariado, BV y modelo bivariado con repetibilidad, BVR). Las características usadas como correlacionadas en los modelos bivariados fueron: producción de leche (PL), porcentaje de proteína (PRO) y porcentaje de grasa (GRA). Las soluciones de las ecuaciones de los modelos lineales mixtos fueron obtenidas mediante el método de máxima verosimilitud restricta libre de derivadas. En esta investigación se determinó que los modelos bivariados (BV y BVR), mejoraron la exactitud de los EBVs en todas las características reproductivas evaluadas en 3 puntos porcentuales en Holstein y 2 puntos porcentuales en Jersey, comparados con los modelos univariados (UV y UVR). Las evaluaciones genéticas realizadas mediante análisis bivariados, incrementan la exactitud de los valores genéticos en características reproductivas (IEP, DA, NSC y TC), cuando se usan características productivas (PL, PRO y GRA) como correlacionadas.

Genetic Evaluation Using Direct and Correlated Traits in Colombian Holstein and Jersey cattle

\section{SUMMARY}

\section{ADDITIONAL KEYWORDS}

Accuracy.

Animal model.

Estimated breeding value.

INFORMATION

Cronología del artículo.

Recibido/Received: 23.12.2016

Aceptado/Accepted: 01.09.2018

On-line: 15.10 .2018

Correspondencia a los autores/Contact e-mail:

¡c.zambrano@uninavarra.edu.co
The aim of this study was to estimate breeding values of fertility traits, using univariate and bivariate models in Holstein and Jersey cattle, in Antioquia, Colombia. The information was obtained from 92 dairy herds, located in 18 municipalities of Antioquia. The fertility traits evaluated were: calving interval (IEP), days open (DO), number of services per conception (NSC) and conception rate (TC). The estimated breeding values (EBV) for fertility traits were calculated using two types of univariate animal model (univariate model UV and univariate model with repeatability UVR) and two types of animal bivariate models (bivariate model BV and BVR bivariate model with repeatability). The traits used as correlated in bivariate models were: milk production (PL), protein percentage (PRO) and fat percentage (GRA). The solutions of the equations of the linear mixed models were obtained through the maximum likelihood derivative free restricted method. In this investigation, was determined that the bivariate models (BV and BVR), improve the accuracy of EBVs in all reproductive traits by 3 and 2 percentage points in Holstein and Jersey respectively compared with univariate models (UV and UVR). The results obtained in this study show that the genetic evaluations performed through bivariate analysis, increase the accuracy of EBVs in fertility traits (IEP, DA, NSC and TC), when production traits (PL, PRO and GRA) were used as correlated.

\section{INTRODUCCIÓN}

El aumento de la producción de leche ha sido uno de los objetivos más importantes en los hatos de lechería especializada en muchos países del mundo. Sin embargo, la producción de altos volúmenes de leche por vaca, ha incrementado los problemas en la fertilidad de los animales, como consecuencia del fuerte antagonismo entre producción y reproducción, ocasionando pérdidas económicas por el bajo desempeño reproductivo de las vacas, reflejado en las bajas tasas de fertilidad, intervalo entre partos prolongado, pocos terneros por vaca por año, elevados costos de reemplazo, menor vida productiva, mayor número de servicios por concepción, entre otros (Kadarmideen et al. 2003, p.184; Miglior et al. 2005, p.1255; Camargo 2012, p.17).

Esta problemática ha obligado a que, en los últimos años, muchos países hayan incluido características de fertilidad en sus esquemas de selección, a pesar de que estas características sean difíciles de evaluar, siendo una de sus limitaciones su baja heredabilidad (Wall et al. 2003, p.4099; Sbardella \& Gaya 2010, p.159). Como consecuencia, la selección directa de estas característi- 
cas puede ser ineficiente y sesgada, principalmente en vacas y toros jóvenes (Walsh et al. 2011, p.128; Jamrozik et al. 2005, p.2199); por lo que, algunos autores, sugieren realizar evaluaciones genéticas conjuntas entre características de fertilidad y características productivas, usando modelos multivariados para mejorar la exactitud de los valores genéticos y disminuir el sesgo de las evaluaciones genéticas (Schaeffer 1984, p.1579; Kadarmideen et al. 2003, p.184). La ventaja de usar modelos multivariados es el aumento en la cantidad de información al considerar asociaciones entre variables, con lo que se logra mejorar la estructuración de los datos, tanto para efectos fijos como para efectos aleatorios (Pollak \& Quaas 1981, p.258; Pollak et al. 1984, p.1590).

En Colombia, el mejoramiento genético en ganado lechero es muy limitado, como consecuencia de la falta de financiación por parte de las entidades gubernamentales y por ende, la información fenotípica registrada de los animales es muy escasa y las evaluaciones genéticas para características productivas, lineales, sanitarias y de fertilidad son muy esporádicas (Solarte \& Zambrano 2012, p.540; Zambrano et al. 2014, p.496). Por lo tanto, es muy importante desarrollar investigaciones que involucren evaluaciones genéticas para estas características en condiciones propias del trópico alto colombiano. El objetivo de este estudio fue estimar valores genéticos en características reproductivas mediante modelos univariados y modelos bivariados en ganado Holstein y Jersey de Antioquia, Colombia.

\section{MATERIAL Y MÉTODOS}

Esta investigación fue aprobada y avalada por el Comité de Ética en Investigación de la Universidad Nacional de Colombia sede Medellín, considerando que cumple con los estándares establecidos para este tipo de estudio (Número de la carta de aprobación: CEMED-015 mayo, 2012).

La presente investigación fue realizada con información tomada de 85 hatos de raza Holstein y 7 hatos de raza Jersey, ubicados en 18 municipios del Departamento de Antioquia (Colombia). Toda la información reproductiva de cada hato (fechas de parto, fechas de secado, fecha de inseminación, fechas de detección de preñez, entre otras), fue obtenida de los registros históricos, libretas y software de manejo ganadero.

Las características reproductivas incluídas en esta investigación y el número de registros para Holstein y Jersey respectivamente fueron: intervalo entre partos (IEP, $n=10.303, n=723$ ), número de servicios por concepción (NSC, $\mathrm{n}=12.883 \mathrm{n}=879$ ), días abiertos (DA, $\mathrm{n}=10.156, \mathrm{n}=715$ ) y tasa de concepción (TC, $\mathrm{n}=12.883$, $\mathrm{n}=879$ ). Esta última fue definida como el porcentaje de éxito de que las inseminaciones llevadas a cabo fueron satisfactorias, y fue calculada como el inverso del número de inseminaciones hasta la concepción en porcentaje [TC $\left.=(1 / \mathrm{NSC})^{*} 100\right]$, como lo describe Weller \& Ezra (1997, p.587). Las características produc- tivas y el número de registros para Holstein y Jersey respectivamente fueron: producción de leche medida en litros/lactancia (PL, n=7.494, n=734), porcentaje de proteína ( $\mathrm{PRO}, \mathrm{n}=5.526, \mathrm{n}=383$ ) y porcentaje de grasa de la leche (GRA, n=5.517, n=374). Los datos extremos considerados fisiológicamente anormales, datos registrados erróneamente o datos que generaban sospecha de su invalidez, fueron eliminados. Los animales que fueron incluidos en la matriz de parentesco para la raza Holstein fueron 7937, de los cuales 755 fueron madres, 593 fueron padres (con un promedio de 8 hijas/toro). Para la raza Jersey, el número de animales incluidos en la matriz de parentesco fue de 833 de los cuales 93 fueron sementales (con un promedio de 6 hijas/toro) y 108 madres. El número de lactancias por vaca fue de 2.4 para la raza Holstein y 1.6 para la raza Jersey.

Para cada una de las características fue evaluada la normalidad de los datos (pruebas de Anderson Darling y Kolmogorov-Smirnov) y la homogeneidad de varianzas (prueba de Levene), usando los procedimientos UNIVARIATE y GLM de SAS (2006) respectivamente.

La estimación de valores genéticos para características reproductivas fue realizada empleando cuatro tipos de modelo animal (Mrode \& Thompson 2005, p.39-119), como se describe a continuación:

I) Modelo animal univariado (denotado como UV), se describió de la siguiente manera:

$$
y=X b+Z a+e
$$

Donde: $\mathrm{y}=$ vector de observaciones para una característica (DA, IEP, NSC y TC), b = vector de efectos fijos dentro de los cuales fueron incluidos: el efecto fijo hato, el efecto fijo número de parto (vacas entre 1 y 8 partos en Holstein y entre 1 y 4 partos en Jersey) y el efecto fijo del grupo contemporáneo (municipioaño de parto-época de parto), dentro del cual fueron conformados 123 grupos en Holstein y 31 grupos en Jersey con al menos 5 registros cada uno. Los efectos aleatorios incluidos en el modelo fueron; $\mathbf{a}=$ vector del efecto aleatorio genético aditivo y $\mathbf{e}=$ residual y $\mathbf{X}$ y $\mathbf{Z}=$ matrices de incidencia que relacionan los registros con los efectos fijos y aleatorios. Se asume que los efectos aleatorios genético aditivo (a) y del residual (e) para la $i$ th característica se distribuyen independientemente con media cero y varianza: $\operatorname{var}(e)=I \sigma_{e}^{2} \mathrm{y}$ $\operatorname{var}(a)=A \sigma_{a}^{2}$, donde A es la matriz de parentesco. Las ecuaciones del modelo mixto para el estimador lineal insesgado (BLUE) de las funciones estimables de b y para el mejor predictor lineal insesgado (BLUP) de a fueron descritas como:

$$
\left[\begin{array}{cc}
X^{\prime} X & X^{\prime} Z \\
Z^{\prime} X & Z^{\prime} Z+A^{-1} \alpha
\end{array}\right]\left[\begin{array}{l}
\hat{b} \\
\hat{a}
\end{array}\right]=\left[\begin{array}{c}
X^{\prime} y \\
Z^{\prime} y
\end{array}\right]
$$


Donde: $\alpha=\sigma_{e}^{2} / \sigma_{a}^{2}$

II) Modelo animal univariado con repetibilidad (denotado como UVR), fue descrito como:

$$
y=X b+W a p+Z a+e
$$

Este modelo además de incluir los efectos considerados en el modelo UV, incluyó el efecto aleatorio de ambiente permanente (ap). En este modelo, la matriz W relaciona los registros con los efectos aleatorios del ambiente permanente y además asume que los efectos aleatorios del ambiente permanente (ap), genético aditivo (a) y del residual (e) para la ith característica se distribuven indenendientemente con media cero y varianza: $\operatorname{var}(a p)=I \sigma_{a p}^{2}, \operatorname{var}(a)=A \sigma_{a}^{2} \mathrm{y} \operatorname{var}(e)=I \sigma_{e}^{2}$ . Las ecuaciones del modelo mixto para el estimador lineal insesgado (BLUE) de las funciones estimables de b y para el mejor predictor lineal insesgado (BLUP) de ap y a fueron descritas como:

$$
\left[\begin{array}{ccc}
X^{\prime} X & X^{\prime} Z & X^{\prime} W \\
Z^{\prime} X & Z^{\prime} Z+A^{-1} \alpha_{1} & Z^{\prime} W \\
W^{\prime} X & W^{\prime} Z & W^{\prime} W+I \alpha_{2}
\end{array}\right]\left[\begin{array}{c}
\hat{b} \\
\hat{a} \\
\hat{a} p
\end{array}\right]=\left[\begin{array}{c}
X^{\prime} y \\
Z^{\prime} y \\
W^{\prime} y
\end{array}\right]
$$

Donde: $\mathrm{I}=$ matriz identidad, $\alpha_{1}=\sigma_{e}^{2} / \sigma_{a}^{2} \mathrm{y}$ $\alpha_{2}=\sigma_{e}^{2} / \sigma_{a p}^{2}$

Modelo animal bivariado (denotado como BV) se describe como:

$$
\left[\begin{array}{l}
y_{1} \\
y_{2}
\end{array}\right]=\left[\begin{array}{cc}
X_{1} & 0 \\
0 & X_{2}
\end{array}\right]\left[\begin{array}{l}
b_{1} \\
b_{2}
\end{array}\right]+\left[\begin{array}{cc}
Z_{1} & 0 \\
0 & Z_{2}
\end{array}\right]\left[\begin{array}{l}
a_{1} \\
a_{2}
\end{array}\right]+\left[\begin{array}{l}
e_{1} \\
e_{2}
\end{array}\right]
$$

Donde: $\mathrm{y}_{\mathrm{i}}=$ vector de observaciones para la $i$ th característica ( $\mathrm{i}=1$ y 2$), 1$ corresponde a una característica reproductiva (IEP, DA, NSC o TC) y 2 una característica productiva (PL, PRO y GRA), obteniendo un total de 12 análisis bivariados, $b_{i}=$ vector de efectos fijos para la $i$ th característica ( $\mathrm{i}=1$ y 2$)$; para la característica 1 , (reproductiva) los efectos fijos se definen igual como en el modelo UV descrito anteriormente, para la característica 2 (productiva), fueron incluidos además de los efectos tenidos en cuenta en el modelo UV, las covariables duración de la lactancia para la característica PL y producción de leche para las características PRO y GRA, $a_{i}=$ vector de los efectos aleatorios genéticos aditivos para la $i$ th característica, $\mathrm{e}_{\mathrm{i}}=$ residual, $\mathrm{X}_{\mathrm{i}}$ y $\mathrm{Z}_{\mathrm{i}}$ $=$ matrices de incidencia que relacionan los registros de la $i$ th característica con los efectos fijos y aleatorios respectivamente.

Se asume que los efectos aleatorios genético aditivo y del residual para la $i$ th característica se distribuyen independientemente con media cero y varianza:

$$
\operatorname{Var}\left[\begin{array}{l}
a_{i} \\
e_{i}
\end{array}\right]=\left[\begin{array}{cc}
G \otimes A & 0 \\
0 & R \otimes I
\end{array}\right]
$$

Donde:

$$
G=\left[\begin{array}{ll}
\sigma_{a 11}^{2} & \sigma_{a 12} \\
\sigma_{a 21} & \sigma_{a 22}^{2}
\end{array}\right] \text { y } R=\left[\begin{array}{ll}
\sigma_{e 11}^{2} & \sigma_{e 12} \\
\sigma_{e 21} & \sigma_{e 22}^{2}
\end{array}\right]
$$

$G=$ matriz de varianzas y covarianzas genéticas aditivas, $\mathrm{I}=$ matriz identidad igual al número de observaciones, $\mathrm{A}=$ matriz de relaciones genéticas aditivas, $\otimes$ = producto directo o de Kronecker, $\sigma_{a 11}^{2}=$ varianza genética aditiva para la característica $1, \sigma_{a 22}^{2}=$ varianza genética aditiva para la característica $2, \sigma_{a 12}$ $=\sigma_{a 21}=$ covarianza genética aditiva entre las característica 1 y $2, R_{0}=$ matriz de varianzas y covarianzas de los residuales, $\sigma_{e 11}^{2}=$ varianza del residual para la característica $1, \sigma_{e 22}^{2}=$ varianza del residual para la característica 2, $\sigma_{e 12}^{e 22}=\sigma_{e 21}=$ covarianza del residual entre las características 1 y 2 .

Las ecuaciones del modelo mixto para el estimador lineal insesgado (BLUE) de las funciones estimables de b y para el mejor predictor lineal insesgado (BLUP) de a fueron definidas como:

$$
\left[\begin{array}{cc}
X^{\prime} R^{-1} X & X^{\prime} R^{-1} Z \\
Z^{\prime} R^{-1} X & Z^{\prime} R^{-1} Z+A^{-1} G^{-1}
\end{array}\right]\left[\begin{array}{c}
\hat{a} \\
\hat{b}
\end{array}\right]=\left[\begin{array}{c}
X^{\prime} R^{-1} y \\
Z^{\prime} R^{-1} y
\end{array}\right]
$$

Donde:

$$
\begin{gathered}
y=\left[\begin{array}{l}
y_{1} \\
y_{2}
\end{array}\right], X=\left[\begin{array}{cc}
X_{1} & 0 \\
0 & X_{2}
\end{array}\right], Z=\left[\begin{array}{cc}
Z_{1} & 0 \\
0 & Z_{2}
\end{array}\right], \mathrm{y} \\
\hat{b}_{i}=\left[\begin{array}{l}
\hat{b}_{1} \\
\hat{b}_{2}
\end{array}\right] \hat{a}_{i}=\left[\begin{array}{l}
\hat{a}_{1} \\
\hat{a}_{2}
\end{array}\right]
\end{gathered}
$$

Modelo animal bivariado (modelo bivariado con repetibilidad, denotado como BVR), se describe como:

$$
\left[\begin{array}{l}
y_{1} \\
y_{2}
\end{array}\right]=\left[\begin{array}{cc}
X_{1} & 0 \\
0 & X_{2}
\end{array}\right]\left[\begin{array}{l}
b_{1} \\
b_{2}
\end{array}\right]+\left[\begin{array}{cc}
Z_{1} & 0 \\
0 & Z_{2}
\end{array}\right]\left[\begin{array}{l}
a_{1} \\
a_{2}
\end{array}\right]+\left[\begin{array}{cc}
W_{1} & 0 \\
0 & W_{2}
\end{array}\right]\left[\begin{array}{l}
a p_{1} \\
a p_{2}
\end{array}\right]+\left[\begin{array}{l}
e_{1} \\
e_{2}
\end{array}\right]
$$

Este modelo además de incluir los efectos y variables considerados en el modelo BV, incluyó el efecto aleatorio de ambiente permanente (ap) para la $i$ th característica, por lo que tiene en cuenta la matriz $W_{i}$ que corresponde a la matriz de incidencia que relaciona los registros de la $i$ th característica con los efectos fijos $y$ aleatorios del ambiente permanente.

Se asume que los efectos aleatorios del ambiente permanente (ap), genético aditivo (a) y del residual (e) 
para la ith característica se distribuyen independientemente con media cero y varianza:

$$
\operatorname{Var}\left[\begin{array}{c}
a_{i} \\
a p_{i} \\
e_{i}
\end{array}\right]=\left[\begin{array}{ccc}
G \otimes A & 0 & 0 \\
0 & Q \otimes I & 0 \\
0 & 0 & R \otimes I
\end{array}\right]
$$

Donde:

$$
\begin{gathered}
G=\left[\begin{array}{ll}
\sigma_{a 11}^{2} & \sigma_{a 12} \\
\sigma_{a 21} & \sigma_{a 22}^{2}
\end{array}\right], Q=\left[\begin{array}{ll}
\sigma_{a p 11}^{2} & \sigma_{a p 12} \\
\sigma_{a p 21} & \sigma_{a p 22}^{2}
\end{array}\right] \mathrm{y} \\
R=\left[\begin{array}{ll}
\sigma_{e 11}^{2} & \sigma_{e 12} \\
\sigma_{e 21} & \sigma_{e 22}^{2}
\end{array}\right]
\end{gathered}
$$

$\mathrm{G}=$ matriz de varianzas y covarianzas genéticas aditivas, $\mathrm{I}=$ matriz identidad igual al número de obser $\mathrm{A}=$ matriz de relaciones genétirac aditivas, $\otimes=$ producto directo o de Kronecker, $\sigma_{a 1}^{2},=$ varianza genética aditiva para la característica $1, \quad \sigma_{a 22}^{2}=$ varianza genética aditiva para la característica 2, $\sigma_{a 12}=\sigma_{a 21}$ ' = covarianza genética aditiva entre las característica 1 y $2, Q=$ matriz de varianzas y covarianzas de los efectos aleatorios del ambiente permanente, $\sigma_{a p 11}^{2}:=$ varianza del ambiente permanente para la característica $1, \sigma_{a p 22}^{2}=$ varianza del ambiente permanente para la característica $2, \sigma_{a p 12}=\sigma_{a p 21}=$ covarianza del ambiente permanente entre las característica 1 y $2, \mathrm{R}$ $=$ matriz de varianzas y covarianzas de los residuales, $\sigma_{e 11}^{2}=$ varianza del residual para la característica 1, $\sigma_{e 22}^{2}=$ varianza del residual para la característica 2, $\sigma_{e 12}=\sigma_{e 21}$ covarianza del residual entre las característica 1 y 2.

Las ecuaciones del modelo mixto para el estimador lineal insesgado (BLUE) de las funciones estimables de b y para el mejor predictor lineal insesgado (BLUP) de a y ap fueron definidas como:

$$
\begin{aligned}
& {\left[\begin{array}{ccc}
X^{\prime} R^{-1} X & X^{\prime} R^{-1} Z & X^{\prime} R^{-1} W \\
Z^{\prime} R^{-1} X & Z^{\prime} R^{-1} Z+k_{1} & Z^{\prime} R^{-1} W \\
W^{\prime} R^{-1} X & W^{\prime} R^{-1} Z & W^{\prime} R^{-1} W+I Q^{-1}
\end{array}\right]\left[\begin{array}{c}
\hat{b}_{i} \\
\hat{a}_{i} \\
\hat{a} p_{i}
\end{array}\right]=\left[\begin{array}{c}
X^{\prime} R^{-1} y \\
Z^{\prime} R^{-1} y \\
W^{\prime} R^{-1} y
\end{array}\right]} \\
& k_{1}=G A^{-1}, y=\left[\begin{array}{l}
y_{1} \\
y_{2}
\end{array}\right], X=\left[\begin{array}{cc}
X_{1} & 0 \\
0 & X_{2}
\end{array}\right], \\
& W=\left[\begin{array}{cc}
W_{1} & 0 \\
0 & W_{2}
\end{array}\right], Z=\left[\begin{array}{cc}
Z_{1} & 0 \\
0 & Z_{2}
\end{array}\right], \hat{b}_{i}=\left[\begin{array}{l}
\hat{b}_{1} \\
\hat{b}_{2}
\end{array}\right], \\
& \hat{a}_{i}=\left[\begin{array}{l}
\hat{a}_{1} \\
\hat{a}_{2}
\end{array}\right], \hat{a} p_{i}=\left[\begin{array}{l}
\hat{a} p_{1} \\
\hat{a} p_{2}
\end{array}\right]
\end{aligned}
$$

Los modelos univariados (UV, UVR) y bivariados (BV, BVR) fueron resueltos usando el software MTDFREML descrito por Boldman et al. (1995), que determina las soluciones para cada efecto fijo (b) y aleatorio (a, ap) de las ecuaciones del modelo mixto, mediante el método de máxima verosimilitud restricta libre de derivadas (Smith \& Graser 1986, p.1156-65). Los análisis bivariados (BV y BVR) fueron realizados entre características correlacionadas con igual número de observaciones.

La exactitud (R) de los valores genéticos fue obtenida a partir de la varianza del error de predicción (PEV) y de la varianza genética aditiva (Mrode \& Thompson 2005, p.50-2). La PEV fue calculada usando el error estándar de predicción (SEP), siendo el PEV igual al cuadrado del SEP $\left(P E V=S E P^{2}=d_{i} \sigma_{e}^{2}\right)$, donde $\mathrm{d}_{\mathrm{i}}$ corresponden a los elementos $C^{22}$ de la diagonal de la matriz inversa generalizada de las ecuaciones del modelo mixto. De esta manera, la exactitud de los valores genéticos fue descrita como:

$$
R=\sqrt{1-P E V / \sigma_{a}^{2}}
$$

La exactitud $\mathrm{R}$ fue determinada directamente, usando el programa MTDFREML.

Los valores genéticos obtenidos para características reproductivas (IEP, DA, NSC y TC), mediante modelos univariados (UV y UVR), fueron comparados con los valores genéticos determinados para las mismas características, pero usando modelos bivariados (BV y BVR). La comparación se realizó usando tres métodos: correlación por rangos de Spearman, correlación de Pearson y regresión lineal simple.

I) Coeficiente de correlación por rangos de Spearman: fue usado para comparar la clasificación o ranking de los animales por sus valores genéticos estimados mediante modelos univariados y modelos bivariados. La ecuación empleada fue la siguiente:

$$
r_{s}=1-\frac{6 \sum D^{2}}{N\left(N^{2}-1\right)}
$$

Donde $\mathrm{D}=$ diferencia entre los correspondientes valores genéticos de orden $(x-y)$ determinados en un mismo animal para una característica reproductiva, $\mathrm{x}=$ valor genético obtenido mediante un modelo univariado (UV o UVR), y = valor genético obtenido mediante un modelo bivariado (BV o BVR), $\mathrm{N}$ = número de parejas $(\mathrm{x}, \mathrm{y})$ y $\mathrm{r}_{\mathrm{s}}=$ coeficiente de correlación de Spearman.

II) Coeficiente de correlación de Pearson: fue usado para determinar la asociación entre los valores genéticos obtenidos mediante modelos univariados y modelos bivariados. La ecuación empleada fue la siguiente:

$$
r_{p}=\frac{\operatorname{COV}(x, y)}{\sigma_{x} \sigma_{y}}
$$


Donde, $\mathrm{r}_{\mathrm{p}}=$ coeficiente de correlación de Pearson $\mathrm{x}=$ valor genético para una característica reproductiva obtenido mediante un modelo univariado (UV o UVR), $\mathrm{y}=$ valor genético para la misma característica reproductiva obtenido mediante un modelo bivariado (BV o BVR), $\mathrm{COV}(\mathrm{x}, \mathrm{y})=$ covarianza entre las variables (x) y (y), $\sigma_{\mathrm{x}}=$ desviación estándar de la variable $\mathrm{x}, \sigma_{\mathrm{y}}=$ desviación estándar de la variable y.

III) Coeficiente de regresión lineal simple: fue usado para determinar el cambio en magnitud de los valores genéticos obtenidos mediante modelos bivariados y modelos univariados. La regresión lineal fue definida como:

$$
y=b_{0}+b_{1} x+e
$$

Donde $\mathrm{x}=$ valor genético para una característica reproductiva obtenido mediante un modelo univariado (UV o UVR), y = valor genético para la misma característica reproductiva obtenido mediante un modelo bivariado (BV o BVR), $b_{1}=$ coeficiente de regresión, $b_{0}$ $=$ intercepto y $\mathrm{e}=$ residual.

\section{RESULTADOS Y DISCUSIÓN}

La exactitud promedio de los valores genéticos en todas las características reproductivas evaluadas (DA, IEP, NSC y TC), usando el modelo UV fue de 0.30 en las dos razas (Holstein y Jersey) y cuando se usó el modelo BV que incluyó las características PL, PRO y GRA como correlacionadas, fueron 0.33 para Holstein y 0.32 para Jersey. La característica reproductiva que presentó mayor exactitud en sus valores genéticos cuando se usó el modelo UV fue TC, con valores de 0.31 y 0.35 en promedio en Holstein y Jersey respectivamente, mientras que la característica que presentó la exactitud más baja fue IEP con valores de 0.28 y 0.25 en Holstein y Jersey respectivamente. Cuando se usó el modelo $\mathrm{BV}$, se obtuvo un incremento promedio de la exactitud de los valores genéticos de 3 puntos porcentuales en todas las características reproductivas en Holstein y 2 puntos porcentuales en Jersey, cuando se comparan con el modelo UV. El mayor incremento en la exactitud se dio en la característica IEP para Holstein, cuando es correlacionada con PL y PRO, pasando de 0.28 a 0.33 en los dos casos. En Jersey, igualmente, el mayor incremento en la exactitud de los valores genéticos se dio en la característica IEP, cuando es correlacionada con PRO, pasando de 0.25 a 0.30 (Tabla I).

Por otra parte, la exactitud promedio de los valores genéticos en todas las características reproductivas (DA, IEP, NSC y TC), usando modelos UVR fue 0.25 en la raza Holstein y 0.30 en la raza Jersey y cuando se usó el modelo BVR, que incluyó las características PL, PRO y GRA como correlacionadas, la exactitud promedio fue 0.29 para Holstein y 0.32 para Jersey. Las características reproductivas que presentaron mayores exactitudes en sus valores genéticos usando modelos
UVR fueron: DA e IEP en Holstein con valores de 0.27 en los dos casos y TC en la raza Jersey, con un valor promedio de 0.35 , mientras que las características que presentaron las exactitudes más bajas en sus valores genéticos fueron TC para Holstein con un valor de 0.21 e IEP en Jersey con un valor de 0.25 . Cuando se usó el modelo BVR para estimar valores genéticos en características reproductivas (DA, IEP, NSC y TC), empleando las características PL, PRO y GRA como correlacionadas, se obtuvo un incremento promedio de la exactitud de 4 puntos porcentuales en todas las características reproductivas, en Holstein y 2 puntos en Jersey, cuando se comparan con las exactitudes obtenidas con el modelo UVR. El mayor incremento en la exactitud lo alcanzan las características DA e IEP en Holstein, cuando son correlacionadas con PRO y PL respectivamente, pasando de 0.27 a 0.33 en los dos casos. En Jersey, el mayor incremento en la exactitud lo alcanza la característica IEP, cuando es correlacionada con PRO, pasando de 0.25 a 0.30 (Tabla I).

Como es evidente, los modelos bivariados (BV y BVR) aumentaron la exactitud de los valores genéticos en comparación con la exactitud obtenida con los modelos univariados (UV y UVR) en todas las características reproductivas evaluadas (IEP, DA, NSC y TC), usando cualquiera de las características productivas (PL, PRO o GRA), como correlacionada. Pollak et al. (1984, p.1590-95) y Schaeffer (1984, p.1567-80), afirman, que el incremento en la exactitud de los valores genéticos en los análisis bivariados, se explica por el aumento en la cantidad de información al considerar las asociaciones entre las variables y la mejora en la estructura de los datos, tanto para efectos fijos como para efectos aleatorios. Sin embargo, este aumento en la exactitud al evaluar dos características simultáneamente (una de baja heredabilidad y otra de moderada o alta heredabilidad), se da en ventaja principalmente en características de baja heredabilidad en cuanto al aumento de la exactitud de los valores genéticos. En esta investigación las características reproductivas IEP, DA, NSC y TC, que presentaron valores de $h^{2}<0.10$, las cuales fueron favorecidas, cuando fueron evaluadas en conjunto con las características productivas PL, PRO y GRA, que presentaron heredabilidades moderadas y que oscilaron entre 0.25 y 0.40 , según resultados ya publicados (Zambrano et al. 2014, p.495-506). De acuerdo con Schaeffer (1984, p.1567-80), una amplia diferencia en la heredabilidad y una alta correlación genética entre las características evaluadas, reducen la varianza del error de predicción (PEV), y a su vez mejoran la exactitud de los valores genéticos obtenidos mediante modelos bivariados. En resultados ya publicados (Zambrano et al. 2014, p.495-506), se determinó que las correlaciones genéticas entre características reproductivas (IEP, DA, NSC y TC) y características productivas (PL, GRA y PRO), fueron moderadas y altas con valores de magnitud que oscilaron entre 0.40 y 0.80 para la raza Holstein y 0.20 y 0.50 para la raza 
Jersey, siendo valores que mejoran la exactitud de la estimación de valores genéticos mediante el uso de modelos bivariados, como se describió anteriormente.

Por otra parte, cuando se comparan las exactitudes entre modelos univariados (UV y UVR) en la raza Holstein, se determinó una diferencia de 5 puntos porcentuales en promedio para los valores genéticos de todas las características reproductivas evaluadas (IEP; DA, NSC y TC) en favor del modelo UV. De igual manera, cuando se comparan los modelos bivariados (BV y BVR), el modelo BV fue superior en 4 puntos porcentuales con respecto al modelo BVR. Para la raza Jersey, no hubo diferencias en la exactitud de los valores genéticos entre los modelos que incluyen el efecto del ambiente permanente (UVR y BVR) con respecto a los modelos que no incluyen dicho efecto (UV y BV) (Tabla I). El bajo número de lactancias por vaca, que para esta raza fue 1.6 en promedio y el número reducido de animales evaluados $(n=833)$, puede considerarse como la causa de la igualdad en la exactitud de los valores genéticos obtenidas entre los modelos que incluyen la repetibilidad y los que no la incluyen, caso contrario para la raza Holstein con una población 10 veces más grande y con un número de lactancias por vaca de 2.4 para todas las características reproductivas evaluadas, se determinó que la repetibilidad afecta la exactitud de los valores genéticos, reduciendo el sesgo en la estimación de dichos valores. De esta manera se considera que un modelo que no incluyen el efecto del ambiente permanente o que se disponga de un bajo número de registros repetidos por animal o una población reducida de animales, conllevará a una sobreestimación de la varianza genética aditiva, lo que a su vez implica una sobrestimación de los valores genéticos y de sus exactitudes.

En la Tabla II se muestran los resultados de correlación de Pearson, correlación de Spearman y coeficientes de regresión lineal entre los valores genéticos para características reproductivas $\left(\mathrm{EBV}_{\mathrm{IEP}}, \mathrm{EBV}_{\mathrm{DA}}, \mathrm{EBV}_{\mathrm{NSC}}\right.$ y $\mathrm{EBV}_{\mathrm{TC}}$ ) obtenidos mediante los modelos univaria-

Tabla I. Exactitud de los valores genéticos para características reproductivas (DA, IEP, NSC y TC) usando modelos univariados (UV y UVR) y modelos bivariados (BV y BVR) en ganado Holstein y Jersey de Antioquia (Accuracy of genetic values for reproductive traits (DA, IEP, and TC NSC) using univariate models (UV and UVR) and bivariate models (BV and BVR) in Holstein and Jersey cattle of Antioquia)

\begin{tabular}{|c|c|c|c|c|c|c|c|}
\hline \multirow{2}{*}{ Modelo } & \multirow{2}{*}{$\begin{array}{c}\text { Característica } \\
\text { evaluada }\end{array}$} & \multirow{2}{*}{$\begin{array}{l}\text { Característica } \\
\text { correlacionada }\end{array}$} & Holstein & Jersey & \multirow{2}{*}{ Modelo } & Holstein & Jersey \\
\hline & & & $\mathrm{R}$ & $\mathrm{R}$ & & $\mathrm{R}$ & $\mathrm{R}$ \\
\hline \multirow{3}{*}{ UV } & DA & & 0.29 & 0.30 & \multirow{5}{*}{ UVR } & 0.27 & 0.30 \\
\hline & IEP & & 0.28 & 0.25 & & 0.27 & 0.25 \\
\hline & NSC & & 0.30 & 0.29 & & 0.24 & 0.29 \\
\hline & $\mathrm{TC}$ & & 0.31 & 0.35 & & 0.21 & 0.35 \\
\hline \multirow[t]{8}{*}{ Promedio } & & & 0.30 & 0.30 & & 0.25 & 0.30 \\
\hline & DA & \multirow{4}{*}{ PL } & 0.33 & 0.32 & & 0.29 & 0.32 \\
\hline & IEP & & 0.33 & 0.26 & & 0.33 & 0.26 \\
\hline & NSC & & 0.34 & 0.31 & & 0.28 & 0.31 \\
\hline & $\mathrm{TC}$ & & 0.35 & 0.36 & & 0.25 & 0.37 \\
\hline & Promedio & & 0.34 & 0.31 & & 0.29 & 0.32 \\
\hline & DA & & 0.33 & 0.31 & & 0.33 & 0.31 \\
\hline & IEP & PRO & 0.33 & 0.30 & & 0.31 & 0.30 \\
\hline \multirow[t]{8}{*}{ BV } & NSC & rino & 0.32 & 0.32 & BVR & 0.29 & 0.31 \\
\hline & $\mathrm{TC}$ & & 0.32 & 0.36 & & 0.23 & 0.37 \\
\hline & Promedio & & 0.33 & 0.32 & & 0.29 & 0.32 \\
\hline & DA & \multirow{4}{*}{ GRA } & 0.31 & 0.31 & & 0.30 & 0.31 \\
\hline & NSC & & 0.31 & 0.33 & & 0.31 & 0.29 \\
\hline & $\mathrm{TC}$ & & 0.33 & 0.38 & & 0.26 & 0.33 \\
\hline & IEP & & 0.31 & 0.29 & & 0.24 & 0.38 \\
\hline & Promedio & & 0.32 & 0.33 & & 0.28 & 0.33 \\
\hline \multicolumn{3}{|l|}{ Promedio } & 0.33 & 0.32 & & 0.29 & 0.32 \\
\hline
\end{tabular}

$\mathrm{R}$, exactitud de los valores genéticos; DA, días abiertos; IEP, intervalo entre partos; NSC, número de servicios por concepción; TC, tasa de concepción; PL, producción de leche; GRA, porcentaje de grasa; PRO, porcentaje de proteína; UV, modelo univariado; BVR, modelo univariado con repetibilidad; BV, modelo bivariado; BVR, modelo bivariado con repetibilidad. 
dos (UV y UVR) y valores genéticos para las mismas características, pero estimados mediante los modelos bivariados (BV y BVR), usando como características correlacionadas PL, GRA y PRO. Las correlaciones de Pearson fueron mayores de 0.90 en la raza Holstein y mayores de 0.80 en la raza Jersey, mientras que las correlaciones de Spearman fueron mayores de 0.80 en las dos razas. Por su parte, los coeficientes de regresión lineal oscilaron entre 1.00 y 1.02 en Holstein y 0.95 y 1.02 en Jersey. Resultados similares fueron obtenidos cuando se compararon los valores genéticos obtenidos con los modelos UVR y BVR. En este caso, las correlaciones de Pearson fueron altas, mayores de 0.90 en la raza Holstein y mayores de 0.80 en la raza Jersey. Las correlaciones de Spearman igualmente fueron altas en Holstein y Jersey, con valores superiores a 0.84 y 0.75 respectivamente. En cuanto a los coeficientes de regresión, estos oscilaron entre 0.97 y 1.48 en Holstein y 0.92 y 1.07 en Jersey. Estos resultados sugieren que los modelos bivariados alteran ligeramente la magnitud de los valores genéticos predichos y el ranking o clasificación de los animales con respecto a los modelos univariados, a pesar de que los errores de predicción de los modelos tienden a ser similares. Kadarmideen et al. (2003, p.190), determinaron valores de correlación de Pearson y Spearman de 0.79 y 0.71 para IEP y

\begin{tabular}{|c|c|c|c|c|c|c|c|}
\hline \multirow{3}{*}{$\begin{array}{l}\text { Característica } \\
\text { evaluada }\end{array}$} & \multirow{3}{*}{$\begin{array}{l}\text { Característica } \\
\text { correlacionada }\end{array}$} & \multicolumn{3}{|c|}{ HOLSTEIN } & \multicolumn{3}{|c|}{ JERSEY } \\
\hline & & \multicolumn{6}{|c|}{$\mathrm{EBV}_{\mathrm{UV}} ; \mathrm{EBV}_{\mathrm{BV}}$} \\
\hline & & $r_{p}(E E)$ & $r_{s}(E E)$ & $b(E E)$ & $r_{p}(E E)$ & $r_{s}(E E)$ & $b(E E)$ \\
\hline DA & \multirow{4}{*}{ PL } & $0.93(0.002)$ & $0.87(0.002)$ & $1.00(0.005)$ & $0.96(0.002)$ & $0.93(0.002)$ & $1.00(0.009)$ \\
\hline IEP & & $0.91(0.002)$ & $0.86(0.002)$ & $1.00(0.005)$ & $0.99(0.001)$ & $0.99(0.001)$ & $1.02(0.004)$ \\
\hline TC & & $0.93(0.002)$ & $0.90(0.002)$ & $1.02(0.005)$ & $0.99(0.001)$ & $0.98(0.001)$ & $0.99(0.005)$ \\
\hline NSC & & $0.91(0.002)$ & $0.85(0.002)$ & $1.02(0.005)$ & $0.95(0.004)$ & $0.90(0.004)$ & $0.99(0.012)$ \\
\hline DA & \multirow{4}{*}{ GRA } & $0.96(0.001)$ & $0.91(0.001)$ & $1.00(0.003)$ & $0.86(0.009)$ & $0.82(0.009)$ & $0.97(0.020)$ \\
\hline IEP & & $0.95(0.001)$ & $0.90(0.001)$ & $1.00(0.004)$ & $0.82(0.011)$ & $0.81(0.011)$ & $0.95(0.023)$ \\
\hline NSC & & $0.99(0.001)$ & $0.97(0.001)$ & $1.00(0.002)$ & $0.84(0.010)$ & $0.81(0.010)$ & $0.95(0.024)$ \\
\hline TC & & $0.99(0.001)$ & $0.99(0.001)$ & $1.00(0.001)$ & $0.92(0.006)$ & $0.90(0.006)$ & $0.96(0.015)$ \\
\hline DA & \multirow{4}{*}{ PRO } & $0.93(0.002)$ & $0.87(0.002)$ & $1.01(0.004)$ & $0.98(0.001)$ & $0.97(0.001)$ & $0.98(0.006)$ \\
\hline IEP & & $0.91(0.002)$ & $0.84(0.002)$ & $1.01(0.005)$ & $0.89(0.007)$ & $0.87(0.007)$ & $0.97(0.017)$ \\
\hline NSC & & $0.98(0.001)$ & $0.94(0.001)$ & $1.00(0.002)$ & $0.95(0.003)$ & $0.91(0.003)$ & $1.02(0.012)$ \\
\hline \multirow[t]{2}{*}{ TC } & & $0.98(0.001)$ & $0.97(0.001)$ & $1.00(0.003)$ & $0.99(0.001)$ & $0.98(0.001)$ & $1.01(0.006)$ \\
\hline & & \multicolumn{6}{|c|}{$\mathrm{EBV}_{\mathrm{UVR}} ; \mathrm{EBV}_{\mathrm{BVR}}$} \\
\hline DA & \multirow{4}{*}{ PL } & $0.99(0.001)$ & $0.95(0.001)$ & $1.03(0.002)$ & $0.97(0.002)$ & $0.94(0.002)$ & $1.00(0.009)$ \\
\hline IEP & & $0.99(0.001)$ & $0.96(0.001)$ & $1.00(0.001)$ & $0.99(0.001)$ & $0.98(0.001)$ & $1.01(0.005)$ \\
\hline NSC & & $0.96(0.001)$ & $0.91(0.001)$ & $1.12(0.004)$ & $0.95(0.003)$ & $0.90(0.003)$ & $0.95(0.011)$ \\
\hline TC & & $0.91(0.002)$ & $0.87(0.002)$ & $1.14(0.006)$ & $0.99(0.001)$ & $0.98(0.001)$ & $0.98(0.005)$ \\
\hline DA & \multirow{4}{*}{ GRA } & $0.95(0.001)$ & $0.90(0.001)$ & $0.99(0.005)$ & $0.99(0.001)$ & $0.99(0.001)$ & $1.07(0.003)$ \\
\hline IEP & & $0.93(0.001)$ & $0.87(0.001)$ & $1.03(0.004)$ & $0.81(0.012)$ & $0.80(0.012)$ & $0.92(0.023)$ \\
\hline NSC & & $0.95(0.001)$ & $0.90(0.001)$ & $1.08(0.004)$ & $0.81(0.012)$ & $0.76(0.012)$ & $0.96(0.024)$ \\
\hline TC & & $0.97(0.001)$ & $0.96(0.001)$ & $1.05(0.003)$ & $0.92(0.006)$ & $0.90(0.006)$ & $0.96(0.015)$ \\
\hline DA & \multirow{4}{*}{ PRO } & $0.92(0.002)$ & $0.85(0.002)$ & $1.17(0.006)$ & $0.98(0.001)$ & $0.96(0.001)$ & $1.00(0.007)$ \\
\hline IEP & & $0.94(0.001)$ & $0.88(0.001)$ & $0.97(0.004)$ & $0.89(0.007)$ & $0.87(0.007)$ & $0.97(0.017)$ \\
\hline NSC & & $0.99(0.001)$ & $0.98(0.001)$ & $1.13(0.002)$ & $0.94(0.004)$ & $0.89(0.004)$ & $0.94(0.012)$ \\
\hline TC & & $0.99(0.001)$ & $0.99(0.001)$ & $1.48(0.002)$ & $0.98(0.001)$ & $0.98(0.001)$ & $1.00(0.006)$ \\
\hline
\end{tabular}

DA, días abiertos; IEP, intervalo entre partos; NSC, número de servicios por concepción; TC, tasa de concepción; PL, producción de leche; GRA, porcentaje de grasa; PRO, porcentaje de proteína; $r_{p}$, coeficiente de correlación de Pearson; $r_{s}$, coeficiente de correlación de Spearman; b, coeficiente de regresión lineal; EE, error estándar; $E_{B V} V_{U V}$, valor genético obtenido mediante el modelo UV; $E B V_{U V R}$, valor genético obtenido mediante el modelo $\mathrm{UVR}, \mathrm{EBV}_{\mathrm{BV}}$; valor genético obtenido mediante el modelo $\mathrm{BV}$; $\mathrm{EBV}_{\mathrm{BVR}}$, valor genético obtenido mediante el modelo BVR 
0.73 y 0.69 para NSC, cuando fueron evaluadas individualmente y mediante análisis bivariados, usando la característica producción de leche como correlacionada. En este mismo estudio, se obtuvieron valores de correlación de Pearson y de Spearman de 0.97 y 0.96 para IEP y 0.92 y 0.89 para NSC, cuando se evaluaron únicamente los toros. Los autores sugieren que no hay una perfecta similitud entre los métodos, no obstante, en las evaluaciones genéticas de los toros no hay cambios significativos en el ranking ni en la magnitud de los EBVs y por lo tanto muchos toros podrían ser correctamente seleccionados.

Las características reproductivas se caracterizan normalmente por presentar heredabilidades bajas, mientras que las características productivas se caracterizan por presentar heredabilidades medias y altas; además, las asociaciones genéticas de magnitud moderada entre características reproductivas y características productivas conllevan a una situación en la cual su análisis conjunto mediante modelos bivariados es favorable. Dada esta situación, en la presente investigación se determinó un incremento en la exactitud de los valores genéticos para todas las características reproductivas (IEP, DA, NSC y TC), cuando se emplearon modelos bivariados, usando las características productivas PL, PRO y GRA como correlacionadas, en comparación con los modelos univariados. Además, cuando se incluyó el efecto del ambiente permanente en los modelos univariados y bivariados, las exactitudes de los valores genéticos fueron inferiores, comparadas con las obtenidas mediante modelos que no incluyen tal efecto, reduciendo de esta manera el sesgo en la estimación de dichos valores. Por otra parte, de acuerdo con los resultados obtenidos en esta investigación, se considera que no hay una perfecta similitud entre los modelos bivariados (BV y BVR), con respecto a los modelos univariados (UV y UVR), no obstante, se considera que no hay cambios significativos en el ranking ni en la magnitud de los EBVs, por lo que los animales pueden ser seleccionados usando modelos univariados o bivariados, solo que estos últimos mejoran la exactitud de los valores genéticos en características de baja heredabilidad.

\section{AGRADECIMIENTOS}

Los autores agradecen a la Universidad Nacional de Colombia sede Medellín, a la Cooperativa COLANTA LTDA y al Ministerio de Agricultura y Desarrollo Rural de Colombia por la financiación del programa "Evaluación genética de toros en condiciones tropicales", a partir del cual se obtuvieron los resultados presentados en esta investigación.

\section{BIBLIOGRAFÍA}

Boldman, K, Kriese, L, Van Vleck, L, Van Tassell, C, \& Kachman, S 1995, 'MTDFREML: A Set of programs to obtain estimates of variances and covariances', [Programa de computadora], Clay Center (NE), USDA-ARS, USA.

Camargo, O 2012, 'La vaca lechera: Entre la eficiencia económica y la ineficiencia biológica', Archivos de Zootecnia, vol. 61 (R), pp. 13-29. Jamrozik, J, Fatehi, J, Kistemaker, G, \& Schaeffer, L, 2005, 'Estimates of genetic parameters for Canadian Holstein female reproduction traits', Journal of Dairy Science, vol. 88, pp. 2199-208.

Kadarmideen, H, Thompson, R, Coffey, M, \& Kossaibati, M 2003, 'Genetic parameters and evaluations from single and multiple trait analysis of dairy cow fertility and milk productions', Livestock Production Science, vol. 81, pp. 183-95

Miglior, F, Muir, B, \& Van Doormaal, B, 2005, 'Selection indices in Holstein cattle of various countries', Journal of Dairy Science, vol. 88, pp. 1255-63.

Mrode, R \& Thompson, R 2005, 'Linear models for the prediction of animal breeding values', 2sd edn, CABI Publishing, Cambridge, UK.

Pollak, E \& Quaas, R 1981, 'Monte Carlo study of genetic evaluations using sequentially selected records', Journal of Animal Science, vol. 52, pp. 257-64.

Pollak, E, Van der Werf, J, \& Quaas, R 1984, 'Selection bias and multiple trait evaluation', Journal of Dairy Science, vol. 67, pp. 1590-1595.

SAS, 2006, 'Statistical Analysis Systems', [CD-ROM], Versión 9.1, SAS Inst, Inc, Cary, NC, USA.

Sbardella, M \& Gaya, L 2010, 'Unfavourable side implications of animal breeding in Livestock species: A review', Archivos de Zootecnia, vol. 59 , pp. 157-68.

Schaeffer, L 1984, 'Sire and cow evaluation under multiple trait models', Journal of Dairy Science, vol. 67, pp. 1567-1580.

Smith, S \& Graser, H 1986, 'Estimating variance components in a class of mixed models by restricted máximum likelihood', Journal of Dairy Science, vol. 69, pp. 1156-65.

Solarte, C \& Zambrano, G 2012. 'Characterization and genetic evaluation of Holstein Cattle in Nariño, Colombia', Revista Colombiana de Ciencias Pecuarias, vol. 25, pp. 539-47.

Wall, E, Brotherstone, S, Woolliams, J, Banos, G, \& Coffey, M, 2003, 'Genetic evaluation of fertility using direct and correlated traits', Journal of Dairy Science, vol. 86, pp. 4093-102.

Walsh, S, Williams, E, \& Evans, A 2011 1, 'A review of the causes of poor fertility in high milk producing dairy cows', Animal Reproduction Science, vol. 123, pp. 127-38.

Weller, J \& Ezra, E 1997, 'Genetic analysis of somatic cell concentration and female fertility of Israeli Holsteins by the individual animal model', Journal of Dairy Science, vol. 80, pp.586-93.

Zambrano, J, Rincón, J, \& Echeverri, J 2014, 'Genetic parameters for production and reproduction traits in Colombian Holstein and Jersey cattle', Archivos de Zootecnia, vol. 63, no. 243, pp. 495-06. 